\title{
Informational properties of infinite numbers of objects in concurrent discriminations by monkeys
}

\author{
F. ROBERT TREICHLER \\ Kent State University, Kent, Ohio \\ and \\ THOMAS V. PETROS \\ University of North Dakota, Grand Forks, North Dakota
}

\begin{abstract}
Eight rhesus monkeys were trained on a counterbalanced series of concurrent, two-choice, discrimination tasks that provided different numbers of correct or incorrect objects as lists of discriminanda. Small, large, or infinite lengths of correct or incorrect object lists were combined in different tasks, and acquisition performances were compared. When tasks had an infinite number of objects in their correct list and a small number (4) in their incorrect list, acquisition entailed significantly less error than was seen when a small number of correct objects was paired with an infinite incorrect list. This pattern of outcomes seemed attributable to novelty preference. However, comparison of error distributions from tasks with infinite list lengths to distributions from analogous tasks with fixed list lengths provided some basis for interpreting the way monkeys integrated information that emerged from the temporally discriminative properties of the tasks. One prospective concern was whether or not these performances represented behaviors like those seen in human cognitive discriminations of frequency of occurrence.
\end{abstract}

Multiple-problem learning procedures have provided valuable techniques for investigating information-processing characteristics from a comparative perspective. An especially important outcome has been noted with monkeys trained on acquisitions of a series of different two-choice discrimination problems. The attendant development of improved facility in problem solution with exposure to a succession of problems has provided evidence for the now classic phenomenon of learning sets (Harlow, 1949), and the issue has received continuing consideration in applications and modifications of this basic multiple-problem test (e.g., Rumbaugh \& Pate, 1984).

Another distinctive variant of multiple-problem learning has entailed the acquisition of several different problems with their trials intermixed within a training session (concurrently); under this procedure, monkeys have demonstrated the capacity to retain information about very large numbers of objects and their reward values. For example, after acquiring 124 problems as a retention pool, monkeys were $90 \%$ correct on one-trial memory tests conducted after a period of as many as 90 days (Treichler, 1984). Attempts to define the nature of this retained information have suggested that solutions of this multipleproblem task could be characterized best in terms of the maintenance of a list of correct objects. This property of

Correspondence concerning this article should be addressed to F. Robert Treichler, Department of Psychology, Kent State University, Kent, OH 44242. list maintenance in monkeys has been demonstrated most convincingly when a relatively large number of objects have been paired in two-choice training trials with objects from a shorter list of alternatives (Treichler, Wetsel, \& Lesner, 1977). This procedure has allowed withinsubject comparisons of learning when tasks have entailed lists of relatively few correct and many incorrect (4-16) versus many correct and few incorrect (16-4) objects. The outcomes have shown that for subjects to achieve acquisition criteria on these two tasks, similar amounts of training were required, and thus the tasks were apparently equally difficult. One interpretation of such nondifferential difficulty suggests that solutions always entail the acquisition and retention of the short (4-object) list and, subsequently, either the choice or the exclusion from choice of those 4 objects when small or large sets, respectively, are correct. Although that tactical approach seems efficient, analyses of the monkeys' patterns of errors have indicated that all acquisitions were really characterized by learning the correct list of objects, even though this might be four times longer than the incorrect list. This propensity for preferential use of information about correct rather than incorrect alternatives also seems to be shared with human subjects in both concept formation (Hovland \& Weiss, 1953) and discrimination learning (Walk \& Saltz, 1965) tasks.

In the present investigation, we attempted to provide optimal conditions for monkeys to solve a concurrent task through the selective use of information about incorrect objects. To that end, conditions were imposed wherein 
either a few objects (4) were correct and appeared in twochoice combinations with an infinite number of incorrect objects or the few objects were incorrect and paired with an infinite number of correct objects. The latter procedure represented an attempt to require learning and retention of a relatively short list of incorrect stimuli. Acquisition in the tasks with infinite numbers of correct or incorrect objects was also compared with acquisition in the more typical limited-set-size tasks $(4-16,16-4)$ in our previous studies (Treichler \& Petros, 1986; Treichler et al., 1977). Thus, in the present study, we wanted to evaluate multiple-problem learning when tasks entailed a solution different from retaining the physical properties of the objects that constituted correct lists. Although correct list retention seemed to underlie previous demonstrations of concurrent performance, we intended to induce animals to retain the incorrect alternatives.

Speculative outcomes included several prospects. Animals might: (1) be unable to solve tasks with infinite numbers of objects, especially when these constituted the correct choices, (2) perform acquisitions by retaining information about a small set of incorrect objects that were excluded from choice, or (3) base their choices on some property different from the physical characteristics of intratrial stimulus objects-for example, they might detect some discriminable property of correct or incorrect objects on the basis of their relative frequencies of appearance.

\section{METHOD}

Eight feral, adult, female rhesus macaques with extensive prior exposure to object-discrimination training were tested on simultaneous two-choice problems in a manual WGTA. All problems presented within a test session were generated as pairs of multidimensionally different, commonly used objects like those in Treichler et al. (1977). The objects were held on a formboard in a manner that required the animals to grasp them directly in order for their displacement to serve as a choice response. Correct and incorrect lists contained 4,16 , or an infinite number of objects, with the latter property imposed by presenting a new, novel object on each trial. One object from a correct and one from an incorrect list were always paired on a trial. The problems presented within a concurrent task were selected to provide either 4 correct objects and an infinite number of incorrect objects $(4-\infty)$ or 4 incorrect objects and infinite numbers of correct objects ( $\infty-4)$. Two other tasks were similarly generated but contained only 16 objects as their limited large lists (i.e., 4-16 or 16-4). Distinctively different sets of objects were used for each of the tasks presented to the animals, but within a particular task condition (e.g., the initial 16-4 task), all subjects saw the sarne object sets (with the same reward values) comprising the discriminanda.

The 8 monkeys were divided into two groups to allow counterbalanced orders of task presentation, and, during the course of the experiment, each animal was tested on eight tasks that entailed infinite numbers of objects. Half the subjects began these tests with a 4- $\infty$ task and half began with the $\infty-4$ task, and in each case this was followed by a second task of the same type. Then, a two-task sequence of the other type was given to complete the counterbalanced order. Subsequently, a similar counterbalanced series of tasks (but with different object problems) was presented again in the opposite order. Table 1 depicts the entire sequence of training on the 12 tasks entailed in the present experiment and indicates that, in addition to the infinity tasks noted above, preliminary and follow-up tests were conducted on 4-16 and 16-4 tasks similar to the ones used in previous studies.
Table 1

Sequence (A) of Various Tasks (B) and Their Associated Error Score Measures (C)

\begin{tabular}{|c|c|c|c|c|c|}
\hline \multirow{2}{*}{$\begin{array}{c}\text { A } \\
\text { Test } \\
\text { Series } \\
\end{array}$} & \multirow{2}{*}{$\begin{array}{c}\text { B } \\
\text { Task } \\
\text { Type } \\
\end{array}$} & & \multirow{2}{*}{$\begin{array}{c}\text { C } \\
\text { Median Errors } \\
\text { to Criterion }\end{array}$} & \multicolumn{2}{|c|}{$\begin{array}{c}\text { D } \\
\text { Overall } \\
\text { Median for Each } \\
\text { Infinity Task }\end{array}$} \\
\hline & & & & $4-\infty$ & $\infty-4$ \\
\hline 1 & $4-167$ & \multirow{2}{*}{ pretest } & 47 & \multirow{6}{*}{$\begin{array}{c}\downarrow \\
\downarrow \\
57.25\end{array}$} & \multirow{6}{*}{$\begin{array}{l}\downarrow \\
\downarrow\end{array}$} \\
\hline 2 & $16-4$ & & 39 & & \\
\hline 3 & $4-\infty$ & & 63.5 & & \\
\hline 4 & $4-\infty$ & & 49 & & \\
\hline 5 & $\infty-4$ & & 36 & & \\
\hline 6 & $\infty-4 !$ & & 25 & & \\
\hline 7 & $4-\infty$ & & 51.5 & $\uparrow$ & \multirow{2}{*}{24.0} \\
\hline 8 & $4-\infty$ & & 27.5 & $\uparrow$ & \\
\hline 9 & $\infty-4$ & & 34.5 & & $\uparrow$ \\
\hline 10 & $\infty-4$ & & 14 & & $\uparrow$ \\
\hline 11 & $4-16$ & \multirow{2}{*}{ posttest } & 50 & & \\
\hline 12 & $16-4$ & & 36 & & \\
\hline
\end{tabular}

Note-Tasks bracketed by dotted lines appeared in counterbalanced order, and those with a solid line appeared sequentially (see procedure). The medians in column D are for the four tests with arrows, respectively.

The daily test session for each subject comprised 32 trials ( 8 times through each short list and its appropriate paired objects), and testing continued until a subject attained an acquisition criterion of 16 (or more) consecutive correct trials. Criterion could be achieved through errorless performance on 4 consecutive runs through the list of 4 correct objects or errorless choice of the 16 novel $(\infty)$ objects that had been paired with 4 consecutive runs through the list of incorrect objects. When the limited large set of 16 was provided, the same criteria were enforced, except that errorless choice of all 16 items in the large correct set was required. Testing was terminated if an animal did not achieve criterion within eight 32-trial sessions, but no animal ever failed to meet this criterion on more than one task. Indeed, it is probably fortunate that animals typically met the acquisition criterion in less than the 8-day limit, because a single infinity task might have required 260 objects for presentation of its full term of testing; if every task had included the maximum amount, the overall number of objects required for presentation of all tasks of the present experiment would have been 2,160 . However, because some tasks were rather readily acquired, and the median number of sessions per task was five, the actual number of objects used in the 12 tasks was slightly less than 1,500.

\section{RESULTS}

\section{Errors-To-Criterion Scores}

Table 1 (column C) displays median errors-to-criterion scores for each of the tasks in the present test series. Because of the small number of subjects and the variability of their performances, nonparametric Wilcoxon comparisons were used to assess the significance of differences between scores on the various tasks. When initial acquisition scores on the 16-4 and 4-16 tasks (Tasks 1 and 2) were compared, no significant differences $(p>05)$ were observed. Because this outcome was the same as that seen in several earlier studies, our premise that these two 
tasks were of approximately equal difficulty seemed to be reaffirmed.

For the tasks that entailed an infinite number of correct or incorrect objects, reductions in error were typically associated with the second of any two consecutive presentations of the same task type (e.g., Task 5, $\infty-4$, and Task $6, \infty-4)$. However, in no case was this difference between immediately adjacent same-type tasks statistically reliable (all $p s>.05$ ). In similar fashion, there were no significant differences when an initial and a subsequent reintroduction of the same task type were compared (e.g., Task 3, 4- $\infty$, and Task 7, 4- $\infty$; all $p$ s $>.05$ ). However, comparisons between the scores on the initial and final (fourth) appearance of each task type suggested some general improvement across the course of intratask presentations. For the 4- $\infty$ task, comparisons between Tasks 3 and 8 yielded significant differences $(T=4, p<.05)$, and similar comparisons for the $\infty-4$ tasks (Task 5 vs. Task 10) yielded a similar pattern but failed to reach significance because of 1 subject's atypical performance $(T=7, p>.05)$.

In order to compare performances on the task types that entailed infinite numbers of objects, the median of the four tests on each task type was computed for each subject. The medians of the 8 subjects' four-test medians are shown in Table 1, column D: $4-\infty$ tests $3,4,7$, and 8 , and $\infty-4$ tests $5,6,9$, and 10 . Subsequently, these medians were compared in a Wilcoxon test that revealed significantly fewer errors-to-criterion on the $\infty-4$ tasks than on the $4-\infty$ tasks $(T=0, p<.02)$.

The overall medians from infinity tasks were also compared with those from the pretest tasks with differential, but finite, numbers of objects. Wilcoxon tests of the differences in errors-to-criterion on the initial 16-4 and the $\infty-4$ tasks indicated that significantly less error $(T=3, p<$ $.05)$ was associated with the $\infty-4$ tasks. When the 4-16 and 4- $\infty$ tasks were similarly compared, significantly greater error $(T=2, p<.05)$ was associated with the 4- $\infty$ tasks. Thus, provision of infinite numbers of incorrect objects appeared to yield a relatively more difficult task, whereas infinite numbers of correct objects yielded reduced task difficulty.

After completion of the infinity tasks, the counterbalanced series of 16-4 and 4-16 tasks was once again presented, and when these scores (Tasks 11 and 12) were compared, significant differences in the direction of greater error on the 4-16 task were observed $(T=0$, $p<.02$ ). However, comparisons of outcomes before and after infinity-task training did not yield reliable differences for either the 4-16 (Tasks 1 vs. 11) or the 16-4 tasks ( 2 vs. 12). An overview of these tests suggested that $\infty-4$ tasks were less difficult than 4- $\infty$ tasks, and exposure to the infinite lists seemed to yield a slight, but reliable, increment in sensitivity to differences in list length on the finite, differential-list-length tasks introduced immediately after acquiring infinity tasks. However, when the same animals were tested on 4-16 and 16-4 tasks in another ex- periment conducted about 3 months after the present one, the two task types did not yield significantly different errors-to-criterion scores.

\section{Ordinal Error Proportion Measures}

Another measure relevant to investigations of tasks with differential list lengths is the proportion of error associated with the various objects that make up the correct or incorrect lists. Both we and others (Bessemer \& Stollnitz, 1971) have noted that individual monkeys in concurrent tasks show marked preferences for some objects and apparent aversions to others (not necessarily the same objects for all subjects). Accordingly, we have measured the relative amounts of error associated with an animal's readily discriminated problems (and objects) and with problems that have generated much error. To derive such intrasubject scores, we divided the number of errors associated with each object in a specific list of correct or incorrect objects by the total number of errors the animal made on that task. Each subject's error proportions were ranked from most to least frequent, and a median of the ordered proportions was computed across subjects. When a 4-object list was considered, differences from chance performance or $25 \%$ error per problem were especially noted. If a 16-item list was under study, comparable proportions were derived by combining the four adjacently ranked sets of 4 objects. By this procedure, Treichler et al. (1977) revealed that the correct objects in 16-4 and 4-16 tasks yielded strikingly similar patterns of ordinal proportions of error. In a 4-16 task, the single most difficult correct object (i.e., the one requiring the most errors to criterion) accounted for about $70 \%$ of the total error, while the least difficult object had little $(1 \%)$ error associated with it. The two intermediate problems yielded error proportions of about $20 \%$ and $10 \%$. An almost identical pattern of error proportions was seen when the correct objects in 16-4 tasks were grouped into quarters (i.e., 4 most difficult, next 4 most difficult, etc.) and their error proportions were compared with the 4-16 error proportions. The close correspondence of these functions has been a major factor in support of the contention that monkeys acquire concurrent tasks by retaining correct object lists despite variation in list length.

Another set of proportion measures aids in the interpretation of the acquisition characteristics seen in unequalset-size tasks. These scores focus on the objects from the incorrect lists, and one might speculate that, if these objects were utilized as the basis for discrimination, then preferences for them or aversions to them should be reflected in differences among the error rates associated with relinquishing selections of these discriminanda. When such error measures were compared in our previous 16-4 tasks, it was found that the proportions associated with the four incorrect objects in 16-4 tasks were $33 \%, 26 \%$, $23 \%$, and $18 \%$ for the most to least frequent error, respectively (Treichler et al., 1977). Because those scores were so closely distributed around chance $(25 \%)$, it seemed fur- 
ther confirmed that, relative to the marked differential proportions of correct-list objects, little attention was afforded to the objects of the small, incorrect set.

In the present study, ordinal error-proportion scores derived from small sets were particularly useful in allowing analysis of the influences of infinite list lengths. Of course, it was not possible to generate error proportion scores for the objects constituting a list of infinite length, but it was possible to analyze the error associated with the four objects that recurred and constituted the correct or incorrect lists with which infinite lists had been paired. Table 2 displays median error proportions associated with the four objects that constituted the small sets in each of the present test phases. Sections $A$ and $C$ show proportions for initial and follow-up finite-list tasks, and section B displays outcomes for the intervening tests that presented infinite numbers of objects. Comparisons of sections $A$ and $C$ show them to be very similar and reasonably like the outcomes of our previous measures of 16-4 and 4-16 performances.

When the error patterns of infinity tasks (section B) were considered, some characteristic properties of the tasks with ever-changing objects tasks were revealed. First, the distributions of error related to the four incorrect objects in the $\infty-4$ tasks showed no differences from the patterns seen on their counterpart tasks with limited list lengths (16-4) that had been administered before or after the infinity tests. Recall that this near-random distribution of error proportion has led us to minimize the role of incorrect objects, and conversely, to emphasize the influence of correct objects in guiding choice. The extension of that interpretation to the present infinity tasks seems warranted. Second, on the 4- $\infty$ tasks, error was distributed a bit more equally among the four correct objects than was typical in the present or previous 4-16 tasks. Wilcoxon comparisons between scores from sections A and B indicated a significantly greater error proportion on the most frequently missed item of the 4-16 task and a significantly smaller error proportion on pooled scores from the two least frequently missed objects of that task (all $p$ s $<.05$ ). The implication of these outcomes was

Table 2

Median Ordinal Proportions of Errors $(\times 100)$ Associated with the Objects Comprising Small (4-Object) Sets (underlined) in Initial Tasks (A), Infinity Tasks (B), and Follow-up (C) Tests

\begin{tabular}{|c|c|c|c|c|c|}
\hline \multirow{3}{*}{$\begin{array}{c}\text { Table } 1 \\
\text { Test } \\
\end{array}$} & \multirow{3}{*}{$\begin{array}{l}\text { Task } \\
\text { Type }\end{array}$} & \multicolumn{4}{|c|}{ Error Frequency } \\
\hline & & most - & & & least \\
\hline & & 1 & 2 & 3 & 4 \\
\hline \multicolumn{6}{|l|}{ A Pre } \\
\hline 1 & 4-16 & 69 & 25 & 4 & 1 \\
\hline 2 & $16-4$ & 39 & 28 & 18 & 15 \\
\hline \multicolumn{6}{|l|}{ B Infinity } \\
\hline $3,4,7,8$ & $4-\infty$ & 47 & 28 & 18 & 6 \\
\hline $5,6,9,10$ & $\infty-\underline{4}$ & 36 & 26 & 21 & 16 \\
\hline \multicolumn{6}{|l|}{ C Post } \\
\hline 11 & $4-16$ & 62 & 26 & 8 & 4 \\
\hline 12 & $\overline{16-\underline{4}}$ & 38 & 27 & 21 & 14 \\
\hline
\end{tabular}

that errors were more equally distributed when an infinite number of incorrect objects were present.

\section{DISCUSSION}

Integrating the information from both the errors-tocriterion measures and the error-proportion patterns of the various tasks in this study is essential to choosing among the alternative interpretations speculated upon in the introduction. Clearly, monkeys were capable of solving tasks with infinite numbers of alternatives serving as either correct or incorrect lists of object discriminanda. The attempt to induce animals to retain information about a small, recurrent list of incorrect objects and, subsequently, to exclude these objects from choice does not appear to have been successful. Inspection of the median error proportions associated with the small, incorrect sets of $\infty-4$ tasks (Table 2, section B) shows them to be nearly identical to those seen on 16-4 tasks. These near-chance proportions imply that the incorrect objects did not serve as exclusive cues for solving the task even in the absence of a specifiable set of correct objects. While their detection as frequently recurrent may have aided the overall task solution, they did not function in the same manner as a small set of correct objects. Contrastingly, the proficiency of performance on $\infty-4$ tasks (Table 1, column C) suggests that these tasks were solved by readily detecting and utilizing some property of the correct objects. Because of the ever-changing characteristics of these objects, the discriminations could not be based on specific physical attributes of the objects in the correct set. Rather, the animals seemed able to detect some abstract property that characterized items associated with reward and came to select such objects after relatively few exposures to the intratask training conditions. The property that seems a likely candidate for providing such a cue is "relative frequency of appearance," with an emphasis on infrequency. This attribute of temporally based information has been termed "novelty" and has sometimes been considered a disruptive influence on nonhuman primate discrimination performance. For example, Harlow (1959) treated novelty preference as a "stimulus-perseveration error factor" that adversely affected acquisition of multiple, serially presented problems. In the usual learning-set procedure, this meant that an undue amount of error was associated with atypical objects appearing among the discriminanda that constituted a series of two-choice problems. Similarly, Moss and Harlow (1947) noted that if a single rewarded (correct) object was presented for five trials, and then this object was paired with a new and different one in a twochoice trial, monkeys consistently chose the novel (and incorrect) object. However, with respect to the present tasks, it can be contended that the property of relative rarity of appearance could operate as a generalized cue to support a strategy for choosing objects that met the criterion of "novel," and that integration of events in time thus allowed detection of infrequency and provided a correct discriminative stimulus $(S+)$ rather than a source of 
error. Although avoidance of the more frequently appearing set of objects might offer an alternative explanation, the pattern of error proportions associated with the $\infty-4$ tasks, by virtue of its similarity to that of the results seen with 16-4 tasks (see Table 2), lent little or no support to such a view.

Another set of comparisons from the present series of concurrent tasks also supported the contention of a distinctive and abstracted property represented by the infrequently appearing objects. Recall that when overall median $\infty-4$ performance was compared with the initial 16-4 performances, less error was seen on the infinity tasks, whereas, conversely, 4- $\infty$ median error was shown to be reliably greater than that required for 4-16 tasks. Accordingly, it appeared that choices based on discerning the infrequency/novelty characteristic were even more rapidly adopted than retaining a list of four specific objects. Furthermore, the presence of an infinite number of incorrect alternatives degraded performance, perhaps because of preferential characteristics of the ever-changing object set (i.e., an instance of novelty operating in the manner of Harlow's "error-factor"). This interpretation is supported by the pattern of differences in error proportions noted when information from the 4-16 task outcomes of Table 2 (section $A$ ) is compared with information from the 4- $\infty$ tasks of Table 2 (section B). As is indicated by those proportion scores, significantly less error was attributable to the single most frequently missed item in the 4- $\infty$ tasks, and greater error was associated with the two least frequently missed items. Accordingly, the relatively greater difficulty of 4- $\infty$ tasks may be attributed to intrusion errors that modified the usual error-reduction process by enticing nonsystematic choice of objects from the infinite, incorrect set.

Although influences like those observed here might be treated as simple effects of novelty preference, we suggest that the procedure of employing novel objects on each trial provides a boundary condition for considering frequency of appearance as a discriminative dimension. Under this procedure, ever-changing stimuli were readily selected if they entailed reward, whereas a novel set of incorrect objects seemed to coax errors. However, providing lists of novel stimuli never prevented monkeys from solving tasks in a manner similar to that seen when recurrent objects served as the discriminanda. Assessing enhancement or impairment of task performance by the frequency cue was not a systematic aim; rather, we asked, if maximal infrequency were provided, how likely was that property to be detected and utilized in various concurrent tasks?

Detection of frequency of occurrence has been considered a meaningful aspect of human memory and information processing (Greene, 1989; Hasher \& Zacks, 1984). The focal issue of the human investigations has typically been whether or not persons "automatically" integrate information about frequency of occurrence. "Automaticity" in this context refers to the prospect that detection and utilization of the frequency cue might be an invariant operating characteristic of the system that en- codes experiences into human memory and that the triggering of this system's effects is "inevitable" (Hasher \& Zacks, 1984). Although the present study was not designed to be a direct test of such automatic processing, some of the present outcomes with monkeys do accord well with criteria that Hasher and Zacks have set forth as indicants of automatic encoding. For example, although performances improved with repeated exposures to a particular disparity-of-presentation condition, these differences were slight and fairly characteristic of a particular condition even in the case of a never-before-experienced event, the appearance of novel stimuli on every trial. Similarly, the monkeys adapted to the requirements of transitions from one task type to another, with little variation from the performance that was appropriate for the set of problems under test. Thus, we found that neither task nor training variations precluded our animals' utilization of the differential frequency cue, and such relationships are among those that proponents of automatic processing often invoke as evidence in support of their view.

Consideration of the full test sequence entailed in this study has suggested that macaque monkeys may employ information about how often objects appear in a multipleproblem task that requires long-term retention. Parkinson and Medin (1983), using a short-term memory task, have also noted that monkeys are capable of detecting frequency-of-appearance characteristics. They used a variant of the delayed-matching-to-sample task, and they have termed the frequency-of-appearance cue an "emerging" attribute of discriminanda. The term seems well chosen, for it implies that, while utilization of differential frequency requires exposure to multiple trials within a task, sensitivity to a dimension based on relative rates of appearance might be a similar, though not necessarily identical, operating characteristic in human and animal information processing. More recently, Macphail and Reilly (1989) have developed an operant technique for pigeons that has also yielded evidence for efficient adoption of discriminations based on frequency of prior presentation in a short-term task. In the present series of concurrent discriminations, interproblem frequency information was shown to be an aid to defining sets of objects that were either correct or incorrect. Coming to choose stimuli that fit an abstracted category, new and novel, was especially readily adopted and did not appear to depend on the usual course of acquiring information about the reward values of list items. Accordingly, we contend that concurrent discrimination entails long-term memory that is based on list learning, but that the characteristics of list membership might not depend on defining the physical properties of the objects that constitute a list. Rather, the categorizing of objects may be supported by what have been termed "emerging" or temporally integrated attributes within the multitrial (and, for us, multiple problem) task. It is suggested that the present performances on concurrent discrimination constitute a further example of the property described by Rumbaugh and Pate (1984) wherein "elements of a problem are no longer just specific stimuli to 
which to respond.' Such behaviors may, in fact, reflect what they term "the higher-order processes of inferences and cognition" and share properties in common with the way a variety of organisms integrate and use information about frequency of occurrence.

\section{REFERENCES}

Bessemer, D. W., \& Stollnitz, F. (1971). Retentions of discriminations and an analysis of learning set. In A. M. Schrier \& F. Stollnitz (Eds.), Behavior of nonhuman primates (Vol. 4, pp. 1-59). New York: Academic Press.

GREENE, R. L. (1989). Negative practice effects on frequency discrimination. American Journal of Psychology, 102, 225-232.

HASHER, L., \& ZACKS, R. T. (1984). Automatic processing of fundamental information: The case of frequency of occurrence. American Psychologist, 39, 1372-1388.

Harlow, H. F. (1949). The formation of learning sets. Psychological Review, 56, 51-65.

HaRLow, H. F. (1959). Learning set and error factor theory. In S. Koch (Ed.), Psychology: A study of a science (Vol. 2, pp. 492-537). New York: McGraw-Hill

Hovland, C. I., \& Weiss, W. (1953). Transmission of information concerning concepts through positive and negative instances. Journal of Experimental Psychology, 45, 175-182.
MacPhall, E. M., \& Reilly, S. (1989). Rapid acquisition of a novelty versus familiarity concept by pigeons (Columba Livia). Journal of Experimental Psychology: Animal Behavior Processes, 15, 242-252.

Moss, E. M., HaRLow, H. F. (1947). The role of reward in discrimination learning in monkeys. Journal of Comparative \& Physiological Psychology, 40, 333-342.

Parkinson, J. K., MEdin, D. L. (1983). Emerging attributes of monkey short-term memory. Journal of Experimental Psychology: Animal Behavior Processes, 9, 31-40.

Rumbaugh, D. M., \& PATE, J. L. (1984). The evolution of cognition in primates: A comparative perspective. In H. L. Roitblat, T. G. Bever, \& H. S. Terrace (Eds.), Animal cognition (pp. 569-587). Hillsdale, NJ: Erlbaum.

Treichler, F. R. (1984). Long-term retention of concurrent discriminations by monkeys. Physiological Psychology, 12, 92-96.

Treichler, F. R., Petros, T. V. (1986). Information about incorrect choices and the transfer of concurrent discriminations by monkeys. American Journal of Psychology, 99, 497-507.

Treichler, F. R., Wetsel, W. C., Lesner, S. A. (1977). Some characteristics of concurrent discrimination and retention by monkeys. Learning \& Motivation, 8, 213-228.

WALK, R. D., SALTZ, E. J. (1965). Discrimination leaming with varying numbers of positive and negative stimuli by children of different ages. Psychonomic Science, 2, 95-96.

(Manuscript received January 5, 1990;

revision accepted for publication November 21, 1990.) 